\title{
An ANFIS Approach for Real Power Transfer Allocation
}

\author{
Hussain Shareef, ${ }^{1}$ Saifulnizam Abd Khalid, ${ }^{2}$ \\ Mohd Wazir Mustafa, ${ }^{2}$ and Azhar Khairuddin ${ }^{2}$ \\ ${ }^{1}$ Faculty of Engineering and Built Environment, The National University of Malaysia, \\ 43600 Bangi, Malaysia \\ ${ }^{2}$ Faculty of Electrical Engineering, Technological University of Malaysia, 81310 Johor, Malaysia \\ Correspondence should be addressed to Saifulnizam Abd Khalid, nizam@fke.utm.my
}

Received 17 June 2010; Accepted 13 September 2010

Academic Editor: Gani Stamov

Copyright (C) 2011 Hussain Shareef et al. This is an open access article distributed under the Creative Commons Attribution License, which permits unrestricted use, distribution, and reproduction in any medium, provided the original work is properly cited.

This paper proposes an adaptive neurofuzzy interface system (ANFIS) approach to identify the real power transfer between generators. Based on solved load flow results, it first uses modified nodal equation method (MNE) to determine real power contribution from each generator to loads. Then the results of MNE method and load flow information are utilized to train the designed ANFIS. It also incorporated an enhanced feature extraction method called principle component analysis (PCA) to reduce the input features to the ANFIS. The 25-bus equivalent system of south Malaysia is utilized as a test system to illustrate the effectiveness of the ANFIS output compared to that of the MNE method. The ANFIS output provides promising results in terms of accuracy and computation time. Furthermore, it can be concluded that the ANFIS with enhanced feature extraction method reduces the time taken to train the ANFIS without affecting the accuracy of the results.

\section{Introduction}

The introduction of electricity privatization becomes an important issue under electric industry restructuring. The aim of this research is to bring transparency and open access to the transmission network. Implementing transparent rules that allocate transmission use fulfill this concept of fairness in the industry. Fairness can only be achieved by adopting a fair and transparent usage allocation methodology acceptable to all parties. In view of market operation, it is vital to know the role of individual generators and loads to transmission wires and power transfer between individual generators to loads. This is necessary for the restructured power system to operate economically, efficiently 
and ensure open access to all system users [1]. Several schemes have been developed to solve the allocation problem in the last few years. Methods based on the $Y$-bus or Z-bus system matrices have recently received great attention since these methods can integrate the network characteristics and circuit theories into line usage and loss allocation. The method reported in [2] is based on Kirchhoff's current law (KCL), equivalent linear circuit that reaches all lines and loads. Based on the stated assumptions, a recursive procedure was used to construct the equivalent circuit for each bus. Moreover, superposition theorem was applied to the bus's equivalent circuit starting from a bus whose injected currents were known. Another circuit concept method was proposed by Chang and $\mathrm{Lu}$ [3]. It was based on the system $Y$-bus and Z-bus matrix modification where, branch current are determined as a function of generators' injected current by using information from the $Z$ bus. Similarly, contribution to bus voltages was computed as a function of each generator current injection by decomposing the network into different networks. Using the computed voltages and currents, the power flowing on the transmission lines were unbundled. It uses approximate formulation to calculate the unbundled loss components. This algorithm utilizes the network decomposition concept as proposed by Zobian and Ilić [4] which determines the use of transmission network by individual bilateral contracts. Teng [5], proposed a systematic method, very similar to that presented in [3], to allocate the power flow and loss for deregulated transmission systems. Using a similar concept, the authors of this paper introduce a modified nodal equation (MNE) method for real and reactive power allocation [6] in which the load buses powers are represented as a function of the generators' current and voltage.

The tracing methods $[1,7-10]$ based on the actual power flows in the network and the proportional sharing principles were effectively used in transmission usage allocation. The methods reported in $[1,9]$ are based on tracing the current and complex power from individual power sources to system loads. Based on solved load flow, the method converts power injections and line flows into real and imaginary current injections and current flows. This method has a clear physical meaning and its results are unique. Bialek in [7] proposed a novel power tracing method. However this method requires inverting a large matrix. Wu et al. [8] proposed graph theory to calculate the contribution factor of individual generators to line flows and loads and the extraction factor of individual loads from line flows and generators, which is theoretically efficient. This method cannot handle loop flows and losses must be removed initially. Reference [11] was based on the concept of generator "domains", "common", and "links". The disadvantage of this method is that the share of each generator in each "common" (i.e., the set of buses supplied from the same set of generators) is assumed to be same. Furthermore, the "commons" concept can lead to problems since the topology of a "common" could radically change even in the case of slight change in power flows. In a related work, a support vector machine (SVM) [12] was applied to estimate the contribution of individual generators to loads in power systems. The SVM gives faster results but the accuracy of the result is not promising.

Therefore in order to obtain fast and accurate results, an adaptive neurofuzzy interface system (ANFIS) approach is proposed in this work. The proposed method considers almost all system variables obtained from load flow solutions as the input features. However, these features are further reduced using the principle component analysis (PCA), to decrease the training time required by the designed ANFIS. The targets of the ANFIS corresponding to the real power transfer allocation results are obtained from MNE method. It is expected that the application of ANFIS can contribute in improving the computation time of real power allocation methodology for deregulated system. 


\section{Modified Nodal Equations Method}

The derivation, to decompose the load real powers into components contributed by specific generators starts with basic equations of load flow. Applying Kirchhoff's law to each node of the power network leads to the equations, which can be written in a matrix form as [1]

$$
I=Y V
$$

where $V$ is a vector of all node voltages in the system, $I$ is a vector of all node currents in the system, and $Y$ is the $Y$-bus admittance matrix.

The nodal admittance matrix of the typical power system is large and sparse, therefore it can be partitioned in a systematic way. Considering a system in which there are $G$ generator nodes that participate in selling power and remaining $L=n-G$ nodes as loads, then it is possible to rewrite (2.1) into its matrix form as

$$
\left[\begin{array}{c}
I_{G} \\
I_{L}
\end{array}\right]=\left[\begin{array}{ll}
Y_{G G} & Y_{G L} \\
Y_{L G} & Y_{L L}
\end{array}\right]\left[\begin{array}{c}
V_{G} \\
V_{L}
\end{array}\right]
$$

Solving (2.2) for $I_{L}$, the load currents can be presented as a function of generators' current and load voltages as

$$
I_{L}=Y_{L G} Y_{G G}^{-1} I_{G}+\left(Y_{L L}-Y_{L G} Y_{G G}^{-1} Y_{G L}\right) V_{L}
$$

Then, the total real power $P_{L}$ of all loads can be expressed as

$$
P_{L}=\operatorname{Re}\left\{V_{L} I_{L}^{*}\right\}
$$

where $(*)$ means conjugate. be found

Substituting (2.3) into (2.4) and solving for $P_{L}$ the relationship as shown in (2.5) can

$$
\begin{aligned}
P_{L} & =\operatorname{Re}\left\{V_{L}\left(Y_{L G} Y_{G G}^{-1}\right)^{*} I_{G}^{*}+V_{L}\left(\left(Y_{L L}-Y_{L G} Y_{G G}^{-1} Y_{G L}\right) V_{L}\right)^{*}\right\} \\
& =\operatorname{Re}\left\{V_{L} \sum_{i=1}^{n G} \Delta I_{L}^{* I_{G}}+V_{L}\left(\left(Y_{L L}-Y_{L G} Y_{G G}^{-1} Y_{G L}\right) V_{L}\right)^{*}\right\},
\end{aligned}
$$

where

$$
\left(Y_{L G} Y_{G G}^{-1}\right)^{*} I_{G}^{*}=\sum_{i=1}^{n G} \Delta I_{L}^{* I_{G}}
$$

where $n G$ is the number of generators.

Now, in order to decompose the load voltage dependent term further in (2.5), into components of generator dependent terms, (2.8) is used. A possible way to deduce load node 
voltages as a function of generator bus voltages is to apply superposition theorem. However, it requires replacing all load bus current injections into equivalent admittances in the circuit. Using a readily available load flow result, the equivalent shunt admittance $Y_{L j}$ of load node $j$ can be calculated using

$$
Y_{L j}=\frac{1}{V_{L j}}\left(\frac{S_{L j}}{V_{L j}}\right)^{*}
$$

where $S_{L j}$ is the load apparent power on node $j$ and $V_{L j}$ is the load bus voltage on node $j$. After adding these equivalences to the diagonal entries of $Y$-bus matrix, (2.1) can be rewritten as

$$
V=Y^{\prime}-1 I_{G}
$$

where $Y^{\prime}$ is the modified $Y$ of (2.1).

Next, adopting (2.8) and taking into account each generator one by one, the load bus voltages contributed by all generators can be expressed as

$$
V_{L}=\sum_{i=1}^{n G} \Delta V_{L}^{* I_{G}}
$$

Now it requires a simple mathematical manipulation to obtain the required relationship as a function of generators dependent terms. By substituting (2.9) into (2.5), the decomposed load real powers can be expressed as

$$
P_{L}=\operatorname{Re}\left\{V_{L} \sum_{i=1}^{n G} \Delta I_{L}^{* I_{G}}+\sum_{i=1}^{n G} \Delta V_{L}^{* I_{G}}\left(\left(Y_{L L}-Y_{L G} Y_{G G}^{-1} Y_{G L}\right) V_{L}\right)^{*}\right\}
$$

This equation shows that the real power of each load bus consists of two terms by individual generators. The first term relates directly to the generators' current and the second term corresponds to their contribution to the load voltages. With further simplification of (2.10), the real power contribution that load $j$ acquires from generator $i$ is

$$
P_{L j}=\sum_{i=1}^{n G} P_{L j i}^{\Delta I_{L}}+\sum_{i=1}^{n G} P_{L j i}^{\Delta V_{L}}
$$

where $P_{L j i}^{\Delta I_{L}}$ is the current dependent term of generator $i$ to $P_{L j}$ and $P_{L j i}^{\Delta V_{L}}$ is the voltage dependent term of generator $i$ to $P_{L j}$.

This allocation method has clear physical meaning as it takes into account the interaction between real and reactive power flows. Vector $P_{L}$ is used as a target in the training process of the proposed ANFIS. 


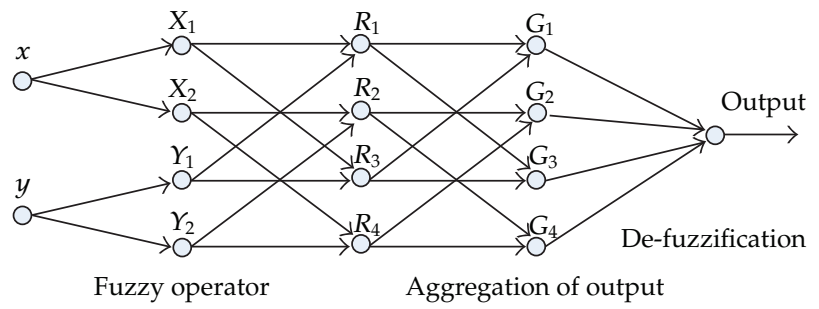

Fuzzification Application method

Figure 1: Basic structure of ANFIS.

\section{Principle of Adaptive Neurofuzzy Inference System (ANFIS)}

Adaptive Neurofuzzy Inference System (ANFIS) is developed from Sugeno-type fuzzy inference system (FIS) for effective data processing. The development is a simple data learning technique by using configuration of neurofuzzy model with hybrid learning rule. FIS processes a given input mapping to get a target output. The ANFIS defines five layers which perform the function of fuzzification of the input values, aggregation of membership degree, evaluation of the bases, normalization of the aggregated membership degree, and evaluation of function output values [13,14].

Basic ANFIS structure consists the five main processing stages illustrated in Figure 1.

The first layer is the input layer which receives input data that are mapped into membership functions so as to determine the membership of a given input. In this fuzzification process the following equations are utilized:

$$
\begin{aligned}
& X_{i}(x)=\frac{1}{\left[1+\left(\left(x-c_{i}\right) / a_{i}\right)^{2}\right]^{b_{i}}}, \quad i=1,2, \\
& Y_{i}(y)=\frac{1}{\left[1+\left(\left(y-c_{i}\right) / a_{i}\right)^{2}\right]^{b_{i}}}, \quad i=1,2,
\end{aligned}
$$

where $X_{i}$ and $Y_{i}$ are fuzzified input values, whereas $a_{i}, b_{i}$, and $c_{i}$ are the parameter sets from the Gaussian input membership function.

The second layer of neurons represents association between input and output, by means of fuzzy rules. Application of fuzzy operators involves the use of the product (AND) to the fuzzified input. Equations (3.2) represent the fuzzy relations obtained from the product fuzzy operators:

$$
\begin{aligned}
& R_{1}=X_{1}(x)+Y_{1}(y), \\
& R_{2}=X_{2}(x)+Y_{2}(y), \\
& R_{3}=X_{3}(x)+Y_{3}(y), \\
& R_{4}=X_{4}(x)+Y_{4}(y) .
\end{aligned}
$$


In the third layer, the output is normalized and then passed to the fourth layer. Here, the activation degree and normalization is implemented by using the following equations

$$
G_{i}=\frac{R_{i}}{\left(R_{1}+R_{2}+R_{3}+R_{4}\right)}
$$

Then the output data are mapped in the fourth layer to give output membership function based on the predetermined fuzzy rules. Aggregation of all outputs is obtained by using (3.4) which is the product of the normalized activation degree and individual output membership function

$$
O_{i}=G_{i}\left(p_{i} x+q_{i} y+r_{i}\right) \quad i=1,2,3,4
$$

where $p_{i}, q_{i}$, and $r_{i}$ are the parameters from the output membership function.

Finally the outputs are summed in the fifth layer to give a single valued output. The ANFIS has the constraint that it only be designed as a single output system and the system must be of unity weights for each rule [15]

$$
O=\sum_{i=1}^{4} O_{i}
$$

\section{Feature Extraction}

An important aspect to be considered for achieving good ANFIS performance is by proper selection and extraction of training data. For large-scale interconnected power systems, the complete state information is too large for any effective ANFIS implementation and therefore, the training data must be reduced to smaller number of useful information [16] using some sort of transformation. In general, the reduced set of features must represent the original set of features, since a loss of information in the reduced set results in loss of performance and accuracy of the ANFIS. The common methods for feature extraction are the linear discriminant analysis (LDA) and principle component analysis (PCA). In this work, PCA is used for feature extraction.

Obtaining the eigenvalues and eigenvectors of the covariance matrices for normal distributions is known as the principal component analysis (PCA). It is a statistical method often used in pattern recognition and data analysis. The goal of PCA is to map vectors having larger dimensional space onto another set of vectors having a smaller dimensional subspace. A brief explanation on calculation of principle components is as follows. Given a data set $X_{l \times m}$, where $l \in \mathfrak{R}^{n}$ represents the number of rows of the data $X$ and $m \in \mathfrak{R}^{n}$ represents the number of input features of the data set. It is possible to calculate the mean value for the input features as

$$
x_{\text {mean }}=\frac{\left(x_{1}+\cdots+x_{m}\right)}{m}
$$




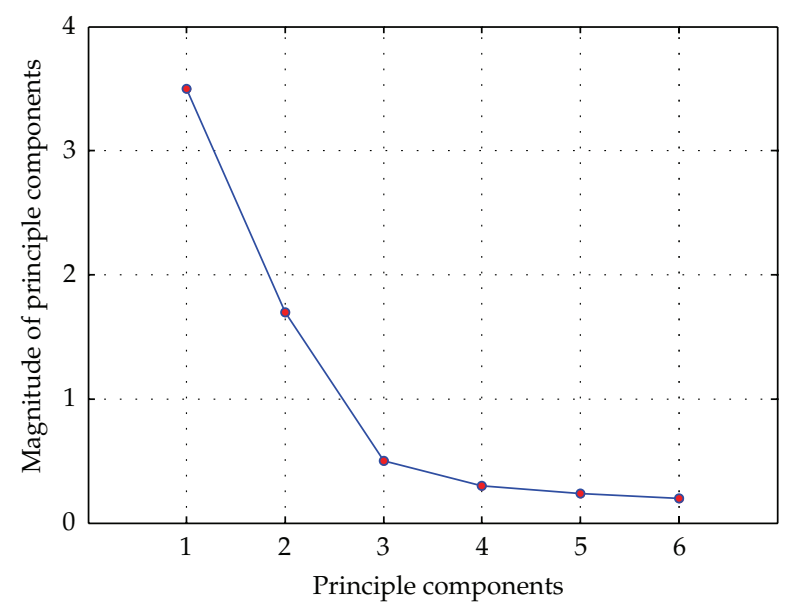

Figure 2: An example of a screen plot showing the principle components and its magnitude.

and subtracting the mean from the original features the covariance matrix can be obtained as

$$
C=\frac{1}{l} \sum_{j=1}^{l}\left(x_{1}-x_{\text {mean }}, \ldots, x_{m}-x_{\text {mean }}\right)^{T}\left(x_{1}-x_{\text {mean }}, \ldots, x_{m}-x_{\text {mean }}\right) .
$$

The final stage of CPA involves the eigenvectors and eigenvalues of the covariance matrix. The new obtained coordinates of the orthogonal projections onto the eigenvectors, are called principle components [16] where the number of principle components is equal to the number of input features. If too many principle components are considered, the transformed input features may include redundant features or if small number of principle components are chosen, they may jeopardize the accuracy of the intelligent system. One method of choosing principle components is by plotting them on a screen plot as shown in Figure 2 [17].

It can be noticed in Figure 2 that there is a "knee" in the plot at the third principle component; therefore according to a popular rule, the number of principle components to be considered should be 3 [17].

\section{ANFIS Design for Real Power Allocation}

In this work, 12 ANFIS blocks are generated and arranged as a hierarchical distribution ANFIS network to obtain real power transfer allocation results for the practical 25-bus equivalent power system of south Malaysia as shown in Figure 3.

This system consists of 12 generators located at buses 14 to 25 , respectively. They deliver power to 5 loads, through 37 lines located at buses 1, 2, 4, 5, and 6, respectively. The data for training is assembled using the daily load curve and performing load flow analysis for every hour of load demand. Similarly the target vector for the training is obtained from the MNE method. Input data $(D)$ for each developed ANFIS contains independent variables such as load bus voltage magnitude $\left(V_{\text {load }}\right)$, real power of load $\left(P_{\text {load }}\right)$, reactive power of load $\left(Q_{\text {load }}\right)$, generator bus voltage magnitude $\left(V_{\text {gen }}\right)$, real power generation $\left(P_{\text {gen }}\right)$, reactive power generation $\left(Q_{\text {gen }}\right)$ corresponding to that particular ANFIS block, average line real power 


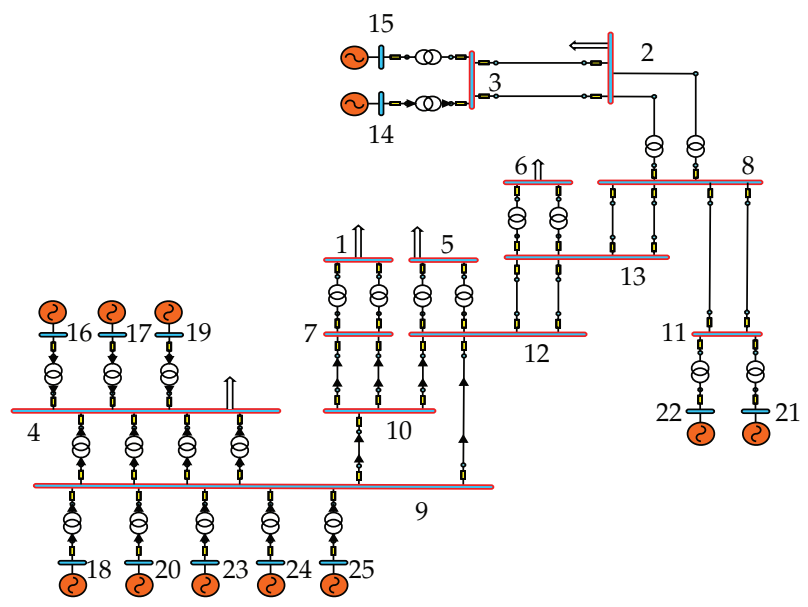

Figure 3: Single line diagrams for the 25-bus equivalent practical power system.

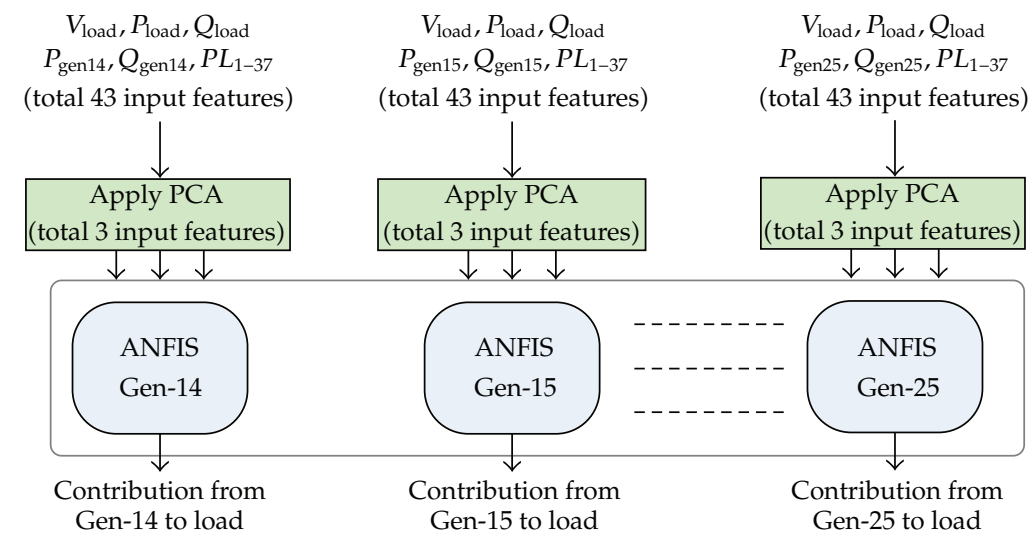

Figure 4: ANFIS design for real power transfer allocation for the 25-bus.

flows $\left(P_{L 1}, P_{L 2}\right.$, to $\left.P_{L 37}\right)$, and the target/output parameter $(T)$ which is the contributions from a generator placed at particular bus to loads. This is considered as a single output from each ANFIS block for real power transfer allocation. Figure 4 shows complete ANFIS design for real power transfer allocation for the 25-bus equivalent practical power system assuming that system topology remains intact. Since PCA uses the eigenvectors of the covariance matrix and it only finds the independent axes of the data, the input data should not vary in abnormally wide range. Therefore for the effective use of PCA, it is assumed that the bus voltages, line flows, are power injections are within the limited range. This assumption is acceptable since the bus voltages, line power flows, generation limits generally varies in a narrow band to maintain voltage, and transient stability of the system. The observed maximum variation in the input samples for bus voltage, load power, generator power, and line power flow, respectively, are $0.037,2.199,0.380$, and 0.535 . Generally it is also noted that the variation in power flow and power injection increases as the load increase according to the daily load curve. 
Table 1: Performance of individual ANFIS blocks.

\begin{tabular}{lccc}
\hline ANFIS block & $\begin{array}{r}\text { Mean error }(\%) \\
\text { ME }(\%)\end{array}$ & $\begin{array}{c}\text { Accuracy }(\%) \\
(100-\operatorname{ME}(\%)\end{array}$ & Prediction time (s) \\
\hline Gen-14 & 0.0136 & 99.9864 & 0.003262 \\
Gen-15 & 0.0136 & 99.9864 & 0.003287 \\
Gen-16 & 0.0136 & 99.9864 & 0.003275 \\
Gen-17 & 0.0048 & 99.9952 & 0.003249 \\
Gen-18 & 0.0133 & 99.9867 & 0.003297 \\
Gen-19 & 0.0216 & 99.9784 & 0.003396 \\
Gen-20 & 0.0042 & 99.9958 & 0.003309 \\
Gen-21 & 0.0261 & 99.9739 & 0.003207 \\
Gen-22 & 0.0652 & 99.9348 & 0.003226 \\
Gen-23 & 0.0018 & 99.9982 & 0.003247 \\
Gen-24 & 0.0502 & 99.9498 & 0.00325 \\
Gen-25 & 0.0432 & 99.9568 & 0.003367 \\
\hline
\end{tabular}

\subsection{Training}

ANFIS is sensitive to the number of input features. Too many input features increasing training time. Therefore number of input features is selected by conducting PCA to eliminate those principle components that contribute less than $2 \%$ to the total variation in the original data set. After the PCA is applied, it is found that the total of input features can be reduced from 43 to only 3 input features without severely affecting the accuracy of the results.

After the reduced input features and target for training data is created, the data $(D$ and $T$ ) is divided into training and test subsets. In this case 168 samples of data are used for the training and another 168 samples are created for testing. Figure 5 shows the performance of the training for individual ANFIS blocks representing each generator. From Figure 5, it can also be seen that the training goal is achieved in 3 epochs with a root mean square error less than $0.2 \times 10^{-4}$.

\subsection{Pretesting and Simulation}

After the ANFIS have been trained, next step is to simulate the ANFIS blocks. The entire test sample data is used in pretesting. After simulation, the obtained result from the trained blocks is evaluated with a linear regression analysis. The regression analysis for the trained ANFIS block that is referred to contribution of generator at bus 14 to loads is shown in Figure 6 . The correlation coefficient, $(R)$ in this case is equal to one which indicates a perfect correlation between MNE method and output of the ANFIS block. The best linear fit is indicated by the solid line, whereas the perfect fit is indicated by the dashed line.

\section{Results and Analysis}

A number of simulations have been carried out to demonstrate the accuracy of the developed ANFIS with the same 25-bus equivalent system of south Malaysia. The scenario here is a decrement by $5 \%$ of the real and reactive load demand from the nominal trained pattern. Besides it also assumed that all generators also decrease their production proportionally 


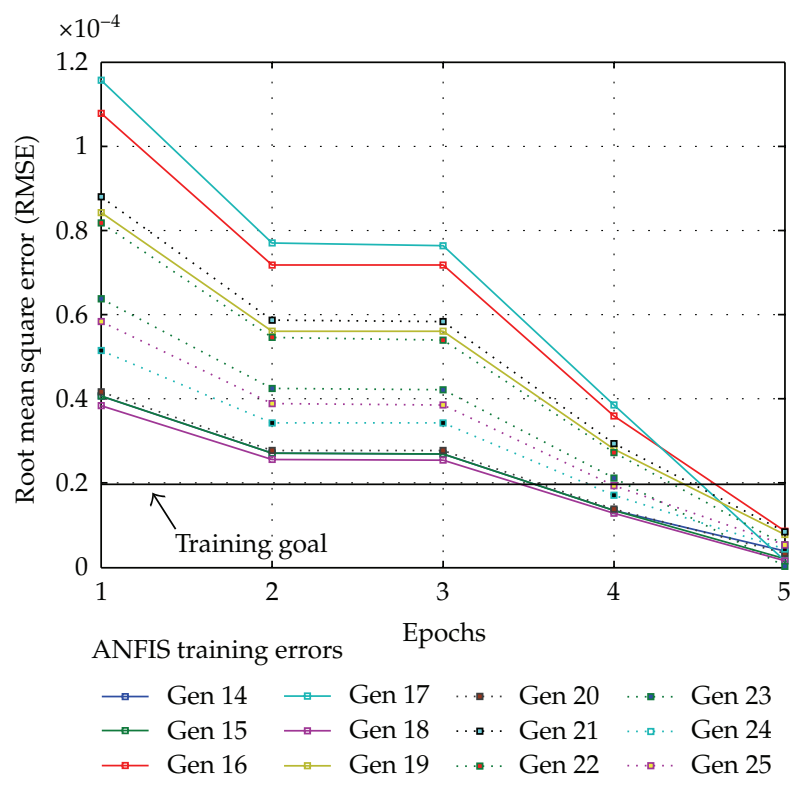

Figure 5: Training curves for individual ANFIS blocks.

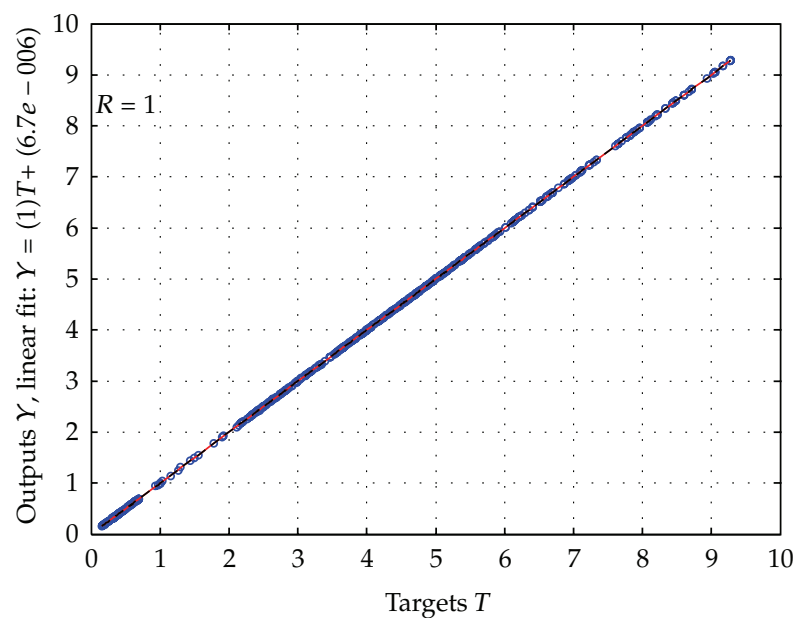

- Data points

- Best linear fit

$---Y=T$

Figure 6: Regression analysis between the ANFIS output and the corresponding target.

according to this variation in the load demands. This assumption is being made to ensure that all real power generation of generator at buses 14 to 25 varies in responding the varying daily load pattern of the loads. Figure 7 shows the real power transfer allocation result for generator located at bus 14 calculated by the ANFIS along with the result obtained through MNE method for loads at buses 1, 2, 4, 5, and 6 for hours 25 to 48 and 121 and 144. 
Table 2: Analysis of real power allocation for the 25-bus equivalent system during hour 33.

\begin{tabular}{|c|c|c|c|c|c|c|c|c|c|c|}
\hline \multirow{3}{*}{$\begin{array}{l}\text { Supplied by } \\
(\mathrm{MW}) \\
\end{array}$} & \multicolumn{10}{|c|}{ Load bus number } \\
\hline & \multicolumn{5}{|c|}{ ANFIS Output } & \multicolumn{5}{|c|}{ MNE Target } \\
\hline & 1 & 2 & 4 & 5 & 6 & 1 & 2 & 4 & 5 & 6 \\
\hline Gen-14 & 0.390 & 4.788 & 3.056 & 4.267 & 5.513 & 0.387 & 4.787 & 3.058 & 4.267 & 5.513 \\
\hline Gen-15 & 0.390 & 4.788 & 3.056 & 4.267 & 5.513 & 0.387 & 4.787 & 3.058 & 4.267 & 5.513 \\
\hline Gen-16 & 0.614 & 7.438 & 41.746 & 6.253 & 8.068 & 0.623 & 7.444 & 41.730 & 6.254 & 8.070 \\
\hline Gen-17 & 0.609 & 7.313 & 40.906 & 6.154 & 7.926 & 0.613 & 7.312 & 40.899 & 6.148 & 7.931 \\
\hline Gen-18 & 0.357 & 4.522 & 2.785 & 3.732 & 4.850 & 0.357 & 4.523 & 2.786 & 3.732 & 4.850 \\
\hline Gen-19 & 0.437 & 5.141 & 28.236 & 4.349 & 5.591 & 0.438 & 5.141 & 28.232 & 4.346 & 5.594 \\
\hline Gen-20 & 0.380 & 4.852 & 2.973 & 3.997 & 5.194 & 0.381 & 4.848 & 2.972 & 3.993 & 5.193 \\
\hline Gen-21 & 0.850 & 9.422 & 6.419 & 7.628 & 10.034 & 0.851 & 9.420 & 6.418 & 7.616 & 10.027 \\
\hline Gen-22 & 0.881 & 9.711 & 6.660 & 7.849 & 10.348 & 0.884 & 9.724 & 6.658 & 7.856 & 10.351 \\
\hline Gen-23 & 0.563 & 7.270 & 4.402 & 5.966 & 7.768 & 0.563 & 7.270 & 4.404 & 5.963 & 7.768 \\
\hline Gen-24 & 0.492 & 6.299 & 3.847 & 5.182 & 6.741 & 0.493 & 6.297 & 3.848 & 5.181 & 6.741 \\
\hline Gen-25 & 0.696 & 8.529 & 5.413 & 7.117 & 9.202 & 0.697 & 8.523 & 5.412 & 7.109 & 9.199 \\
\hline Total & 6.660 & 80.072 & 149.499 & 66.759 & 86.748 & 6.673 & 80.076 & 149.476 & 66.730 & 86.749 \\
\hline Actual & 6.673 & 80.076 & 149.476 & 66.730 & 86.749 & 6.673 & 80.076 & 149.476 & 66.730 & 86.749 \\
\hline
\end{tabular}

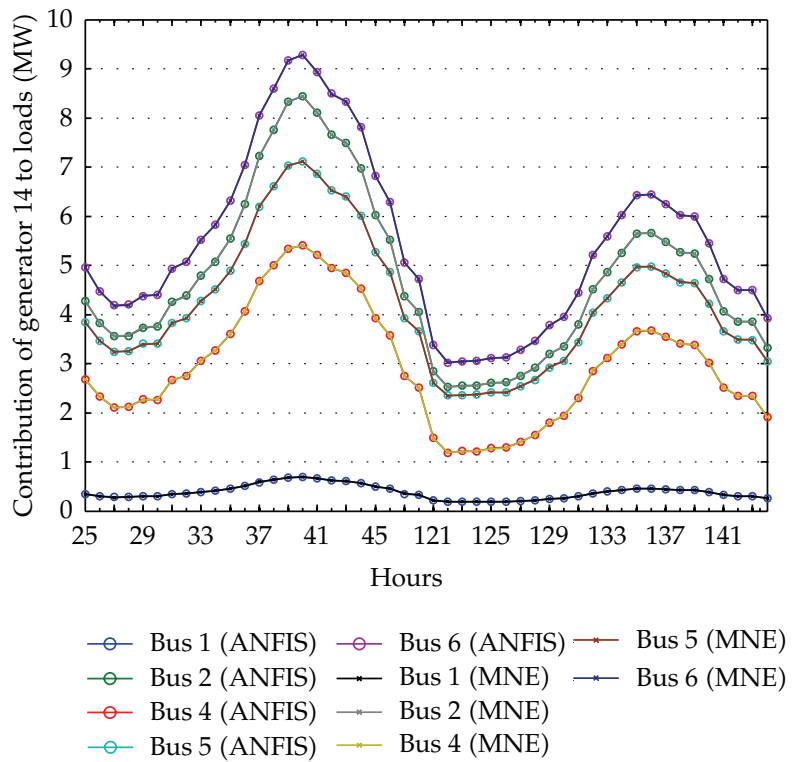

Figure 7: Distribution of real power from generator at bus 14 to loads within hours 25 to 48 and 121 and 144.

Results obtained from the ANFIS are indicated with lines having circles and the solid lines with cross represent the output of the MNE method. From Figure 7, it can be observed that the developed ANFIS can allocate real power transfer between generators and load with very good accuracy, almost $99.95 \%$. In this simulation, ANFIS computes within $39.37 \mathrm{msec}$, whereas the MNE method took $360 \mathrm{msec}$ for the calculation of same real power transfer allocation. Therefore it can be concluded that the ANFIS is more efficient in terms of computation time. Moreover it is worth highlighting that the feature reduction method used 
Table 3: Load flow data for the 25-bus equivalent system at hour 33.

\begin{tabular}{lcccccc}
\hline Bus no. & $\begin{array}{c}\text { Voltage } \\
\text { Magnitude }\end{array}$ & $\begin{array}{c}\text { Angle } \\
\text { (p.u) }\end{array}$ & $\begin{array}{c}\text { Real } \\
\text { (MW) }\end{array}$ & $\begin{array}{c}\text { Reactive } \\
\text { (Mvar) }\end{array}$ & $\begin{array}{c}\text { Real } \\
\text { (MW) }\end{array}$ & $\begin{array}{c}\text { Road } \\
\text { Reactive } \\
\text { (Mvar) }\end{array}$ \\
\hline 1 & 1.0442 & 8.3682 & 0 & 0 & 6.673 & 4.0038 \\
2 & 1.0423 & 7.8052 & 0 & 0 & 80.076 & 24.023 \\
3 & 1.0426 & 7.9571 & 0 & 0 & 0 & 0 \\
4 & 1.0416 & 8.1925 & 0 & 0 & 149.48 & 56.053 \\
5 & 1.031 & 6.1741 & 0 & 0 & 66.73 & 23.355 \\
6 & 1.0335 & 5.6078 & 0 & 0 & 86.749 & 28.027 \\
7 & 1.0455 & 8.4806 & 0 & 0 & 0 & 0 \\
8 & 1.0559 & 8.2109 & 0 & 0 & 0 & 0 \\
9 & 1.0462 & 8.626 & 0 & 0 & 0 & 0 \\
10 & 1.0456 & 8.4878 & 0 & 0 & 0 & 0 \\
11 & 1.0572 & 8.274 & 0 & 0 & 0 & 0 \\
12 & 1.0447 & 8.2158 & 0 & 0 & 0 & 0 \\
13 & 1.047 & 7.7774 & 0 & 0 & 0 & 0 \\
14 & 1.04 & 9.4198 & 31.504 & -2.6853 & 0 & 0 \\
15 & 1.04 & 9.4198 & 31.504 & -2.6853 & 0 & 0 \\
16 & 1.05 & 9.88 & 36.987 & 10.629 & 0 & 0 \\
17 & 1.05 & 9.8454 & 36.229 & 10.606 & 0 & 0 \\
18 & 1.05 & 9.979 & 23.943 & 3.971 & 0 & 0 \\
19 & 1.05 & 9.605 & 24.888 & 8.4126 & 0 & 0 \\
20 & 1.05 & 10.086 & 25.833 & 4.0174 & 0 & 0 \\
21 & 1.153 & 12.721 & 31.504 & 38.05 & 0 & 0 \\
22 & 1.16 & 12.694 & 31.504 & 40.973 & 0 & 0 \\
23 & 1.05 & 10.43 & 39.38 & 5.1706 & 0 & 0 \\
24 & 1.05 & 10.17 & 33.709 & 5.0049 & 0 & 0 \\
25 & 1.055 & 10.594 & 43.16 & 11.322 & 0 & 0 \\
\hline
\end{tabular}

in this work is effective and it does not cause a noticeable error. Table 1 shows the percentage mean error, accuracy, and prediction time taken by individual ANFIS blocks. The percentage mean error, $\mathrm{ME}(\%)$ is calculated from the following equations:

$$
\operatorname{ME}(\%)=\sum_{n=1}^{N} \frac{E_{n}}{N} \times 100
$$

where $E_{n}$ difference between desired output and actual output of test data number $n$ and $N$ is the total number of data.

The allocation of real power to loads using proposed ANFIS on hour 33 out of 168 hours is presented in Table 2 along with the result obtained through MNE method. From Table 2, it can be noted that the result obtained by the ANFIS output in this paper is compared well with the result of MNE method. The difference of real power between generators in both methods is very small which is less than or equal to $0.029 \mathrm{MW}$. The consumer located at bus 4 consumed the highest demand compared to other consumers in this hour. Consequently, the contribution of real power due to generators 16, 17, and 19 located at the same bus provides 
more real power to load at bus 4 by both methods as well. This result also justifies the physical meaning of MNE method as these generators are nearest to load at bus 4 . Moreover, to validate the results, load flow information at hour 33 for the test system are given in Table 3. It can be observed that the sum of the real power contributed by each generator obtained from MNE method and ANFIS is in conformity with the actual power flow.

\section{Conclusion}

In this paper, an ANFIS method has been developed to identify the real power transfer between generators and load. The developed ANFIS adopts real power allocation outputs determined by MNE technique as an estimator to train the ANFIS. The robustness of the proposed method has been demonstrated on the 25-bus equivalent system of south Malaysia. From the results, it can be concluded that the ANFIS output provides the results in a faster and convenient manner with good accuracy. Better computation time is crucial to improve online application. Hence, the proposed method could be adapted to true application of real power allocation and help to resolve some of the difficult real power pricing and costing issues to ensure fairness and transparency in the deregulated environment of power system operation.

\section{Acknowledgments}

The authors wish to acknowledge the Ministry of Higher Education, Malaysia (MOHE) for the financial funding of this project, and Universiti Kebangsaan Malaysia and Universiti Teknologi Malaysia for providing infrastructure and moral support for the research work.

\section{References}

[1] H. Shareef and M. W. Mustafa, "Real and reactive power allocation in a competitive market," WSEAS Transactions on Power Systems, vol. 1, pp. 1088-1094, 2006.

[2] R. Reta and A. Vargas, "Electricity tracing and loss allocation methods based on electric concepts," IEE Proceedings: Generation, Transmission and Distribution, vol. 148, no. 6, pp. 518-522, 2001.

[3] Y.-C. Chang and C.-N. Lu, "Electricity tracing method with application to power loss allocation," International Journal of Electrical Power and Energy System, vol. 23, no. 1, pp. 13-17, 2001.

[4] A. Zobian and M. D. Ilić, "Unbundling of transmission and ancillary services-part I: technical issues," IEEE Transactions on Power Systems, vol. 12, no. 2, pp. 539-548, 1997.

[5] J.-H. Teng, "Power flow and loss allocation for deregulated transmission systems," International Journal of Electrical Power and Energy Systems, vol. 27, no. 4, pp. 327-333, 2005.

[6] H. Shareef, M. W. Mustafa, S. Abd Khalid, A. Khairuddin, A. Kalam, and A. Maung Than Oo, "Real and reactive power transfer allocation utilizing modified Nodal equations," International Journal of Emerging Electric Power Systems, vol. 9, no. 6, pp. 1-14, 2008.

[7] J. Bialek, "Tracing the flow of electricity," vol. 143, pp. 313-320.

[8] F. F. Wu, Y. Ni, and P. Wei, "Power transfer allocation for open access using graph theory fundamentals and applications in systems without loopflow," IEEE Transactions on Power Systems, vol. 15, no. 3, pp. 923-929, 2000.

[9] M. W. Mustafa, H. Shareef, and M. R. Ahmad, "An improved usage allocation method for deregulated transmission systems," in 7th International Power Engineering Conference, IPEC2005, sgp, December 2005.

[10] S. Abdelkader, "Efficient computation algorithm for calculating load contributions to line flows and losses," IEE Proceedings: Generation, Transmission and Distribution, vol. 153, no. 4, pp. 391-398, 2006.

[11] D. K. Ron and A. G. Strbac, "Contributions of individual generators to loads and flows," IEEE Transactions on Power Systems, vol. 12, no. 1, pp. 52-60, 1997. 
[12] H. Shareef, A. Mohamed, S. N. Khalid, M. W. Mustafa, and A. Khairuddin, "Real power transfer allocation utilizing support vector machine," in Proceedings of The International Conference of Electrical Energy and Industrial Electronic Systems (EEIES '09), pp. 1-7, Penang, Malaysia, December 2009.

[13] P. Simon, Oscillatory stability assessment of power system using computational intelligence, Ph.D. thesis, Universit Duishdurg-Essen, Essen, Germany, 2005.

[14] J. R. Jang, "ANFIS: adaptive-network-based fuzzy inference system," IEEE Transactions on Systems, Man and Cybernetics, vol. 23, no. 3, pp. 665-685, 1993.

[15] S. M. Bateni, S. M. Borghei, and D.-S. Jeng, "Neural network and neuro-fuzzy assessments for scour depth around bridge piers," Engineering Applications of Artificial Intelligence, vol. 20, no. 3, pp. 401-414, 2007.

[16] C. A. Jensen, M. A. El-Sharkawi, and R. J. Marks II, “Power system security assessment using neural networks: feature selection using fisher discrimination," IEEE Transactions on Power Systems, vol. 16, no. 4, pp. 757-763, 2001.

[17] B. Scholkopf, A. Smola, and K. R. Muller, Kernel Principal Component Analysis, in Advances in Kernel Methods—SV Learning, MIT Press, Cambridge, Mass, USA, 1999. 


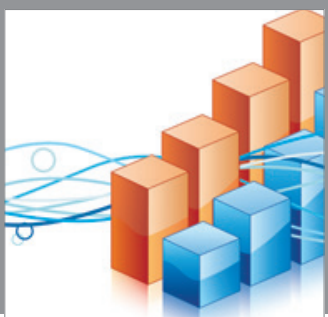

Advances in

Operations Research

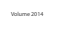

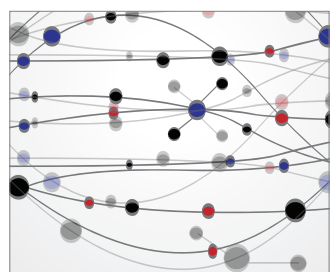

\section{The Scientific} World Journal
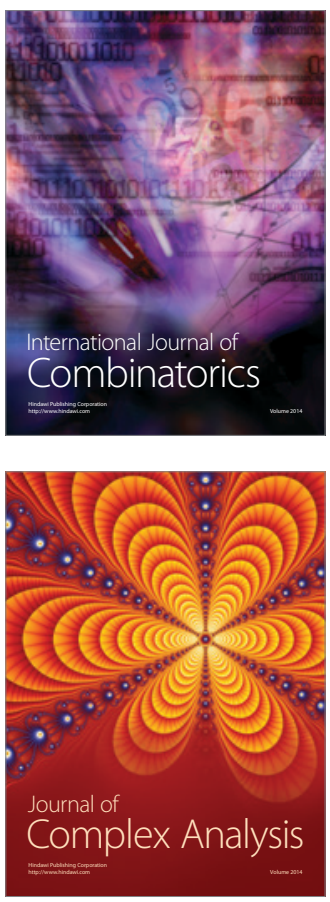

International Journal of

Mathematics and

Mathematical

Sciences
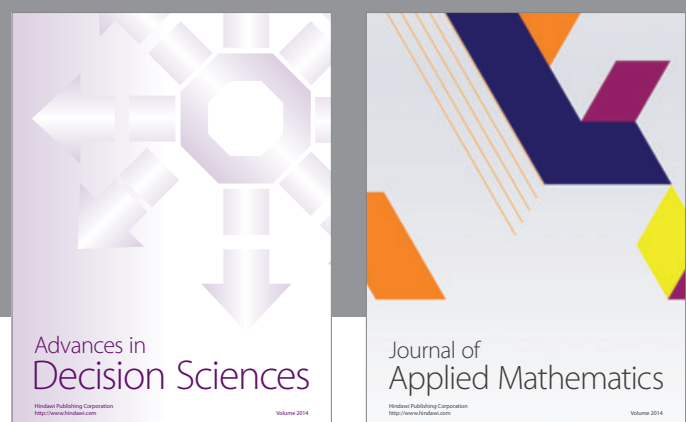

Journal of

Applied Mathematics
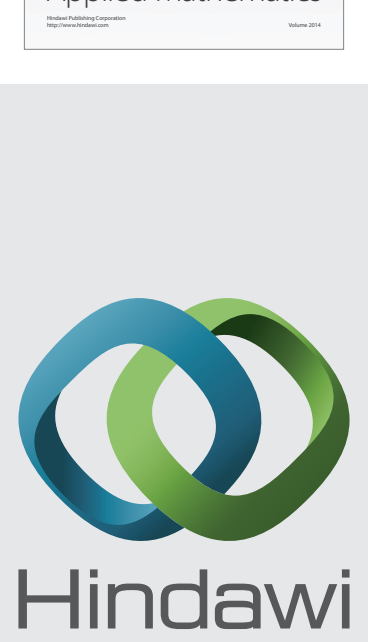

Submit your manuscripts at http://www.hindawi.com
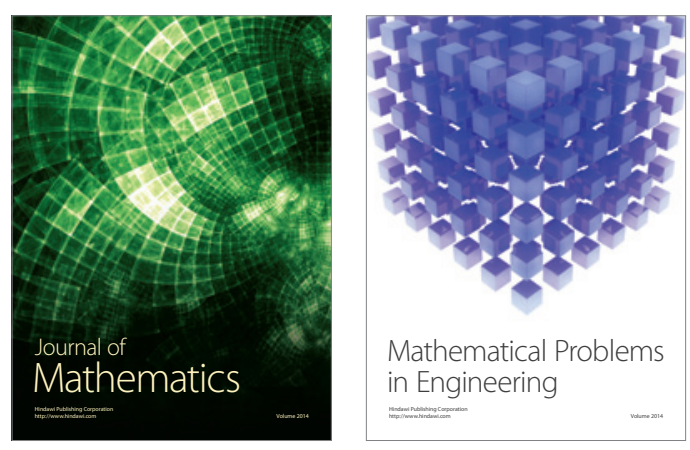

Mathematical Problems in Engineering
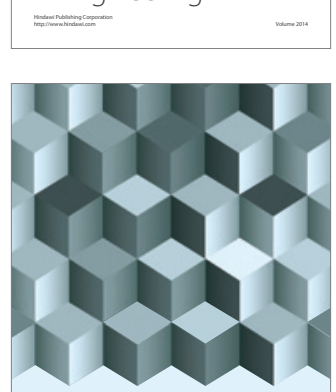

Journal of

Function Spaces
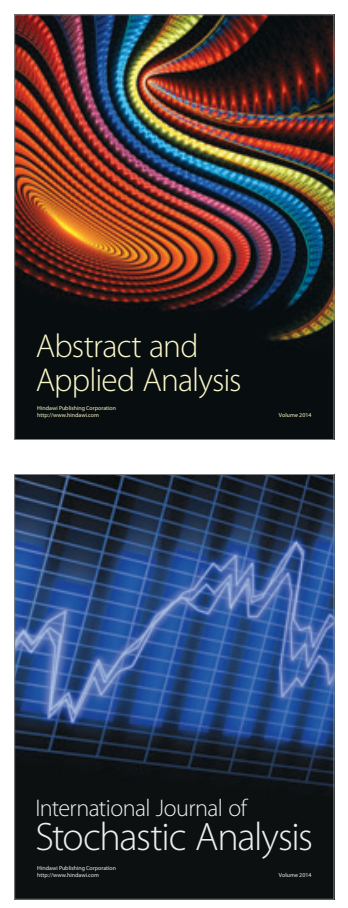

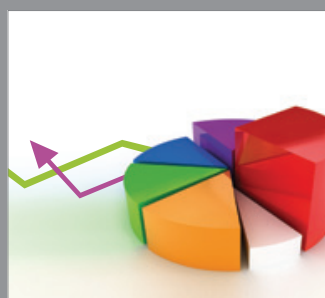

ournal of

Probability and Statistics

Promensencen
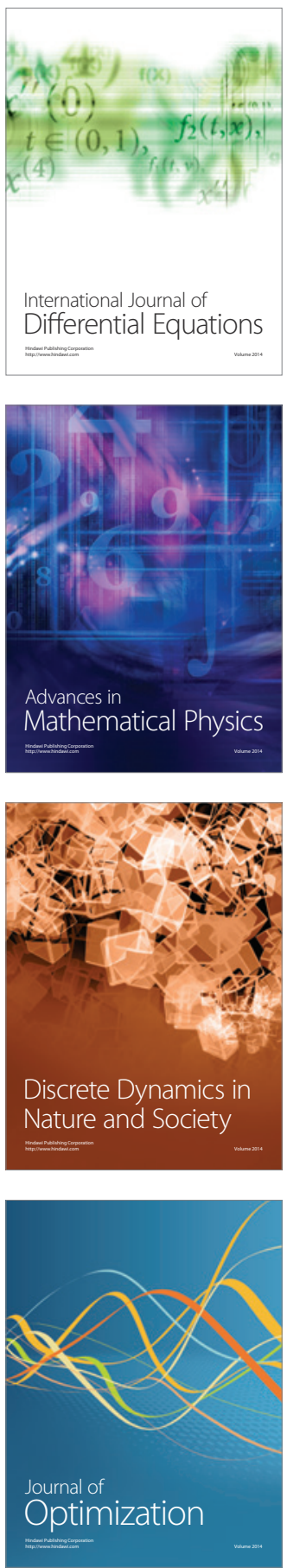